\title{
Research on a Multi-Scale Segmentation Algorithm Based on High Resolution Satellite Remote Sensing Image
}

\author{
Dehui Zhang, Yong Yang \\ School of Information and Electrical Engineering \\ Shenyang Agricultural University \\ Shenyang 110161, China \\ zdh_syit@163.com,yy_syau@163.com
}

\author{
Kai Song, Deyu Zhang \\ School of Information Science and Engineering \\ Shenyang Ligong University \\ Shenyang 110159, China \\ songkai_syit@163.com, zhang_de_yu@163.com
}

\begin{abstract}
A multi-scale segmentation algorithm based on high resolution satellite remote sensing image is proposed in this study. Firstly, scale parameter was set up according to the size of the target object. Then the heterogeneity between adjacent image regions was calculated by the formula. Thereafter, the heterogeneity and scale parameter were compared. If heterogeneity was smaller than the scale parameter, the adjacent image regions were merged and the process of comparing and merging continued; otherwise; if heterogeneity was not smaller than the scale parameter, the process of merging the adjacent image regions ended and image segmentation of remote sensing image completed. Finally, the target objects were divided into single image object. Our experiment shows that this novel algorithm can adjust scale parameter according to the size of the target object, and achieve multi scale segmentation of remote sensing image.
\end{abstract}

Keywords-high resolution satellite remote sensing image; multi-scale; image segmentation; image object

\section{INTRODUCTION}

Remote sensing refers to detect and classify objects remotely, which is a long distance detecting technology without physical contact [1]. Remote sensing technology is firstly applied in investigation and research on various resources in the 1990s in China. With decades of development, this technology has been widely applied in analysis of land utilization and agriculture, investigation of natural disasters, ocean study and military purposes [2]. Multi-scale segmentation of remote sensing image is one of the most important applications of remote sensing technology, which aims to look for the most efficient multi-scale segmentation algorithm [3]. By using the algorithm, the users can adjust scale parameters according to the size of the earth objects [4], and extract earth objects of interest from remote sensing image in a tailored manner. The high resolution satellite remote sensing image has become the major research object of segmentation technology for remote sensing image at present.

There are many commonly used multi-scale segmentation algorithms for remote sensing image, such as algorithm based on region growing, watershed and HIS model [5]. However, the traditional segmentation methods merge the regions by only refereeing to one rule, i.e. similarity of color or spectrum, resulting in either "over-merging" or "deficient merging" [6]. To overcome this shortcoming, we propose a novel multi-scale

In this paper, the research was sponsored by Program for Liaoning hundred million talent project(2014921042). segmentation algorithm based on minimum heterogeneity of neighboring regions in remote sensing image. Specifically, two reference rules including both spectrum feature and shape feature of image are adopted to perform the merging process of the adjacent image regions. Due to the above advantages, our algorithm can accurately merge the adjacent region and achieve good segmentation of remote sensing image.

\section{BASIC IDEAS OF ALGORITHM}

The basic ideas of the multi-scale segmentation algorithm for remote sensing image are as following:

1. Calculate heterogeneity a between one single pixel and its neighboring image region by formula;

2. Set up scale parameter $b$ of remote sensing image. If $a<b$, merge the pixel and its neighboring image region.

3. Repeat calculation and comparison of heterogeneity after merge of image region in step 2 . If $a<b$, merge the pixel and its neighboring image region.

4. If $\mathrm{a} \geq \mathrm{b}$, merging of the neighboring image region ends up and multi-scale segmentation of remote sensing image is completed.

The "multi-scale" in this algorithm is achieved by setting up different scale parameters. The basic ideas of setting up are as the following:

The setup of the scale parameter is directly associated with the size of the earth object. When the earth object is small in size, the scale parameter should be small accordingly. Consequently, the execution times of step 1 and step2 will be decreased and the resulting merged image region will be small. Finally, we obtain properly small earth object. Similarly, when the earth object is large in size, the scale parameter should be large accordingly. Consequently, the execution times of step 1 and step2 will be increased and the resulting merged image region will be large. Finally, we obtain properly large earth object.

\section{FLOW CHART OF THE ALGORITHM}

The flow chart of the multi-scale segmentation algorithm for high resolution satellite remote sensing image is shown in Figure 1. 


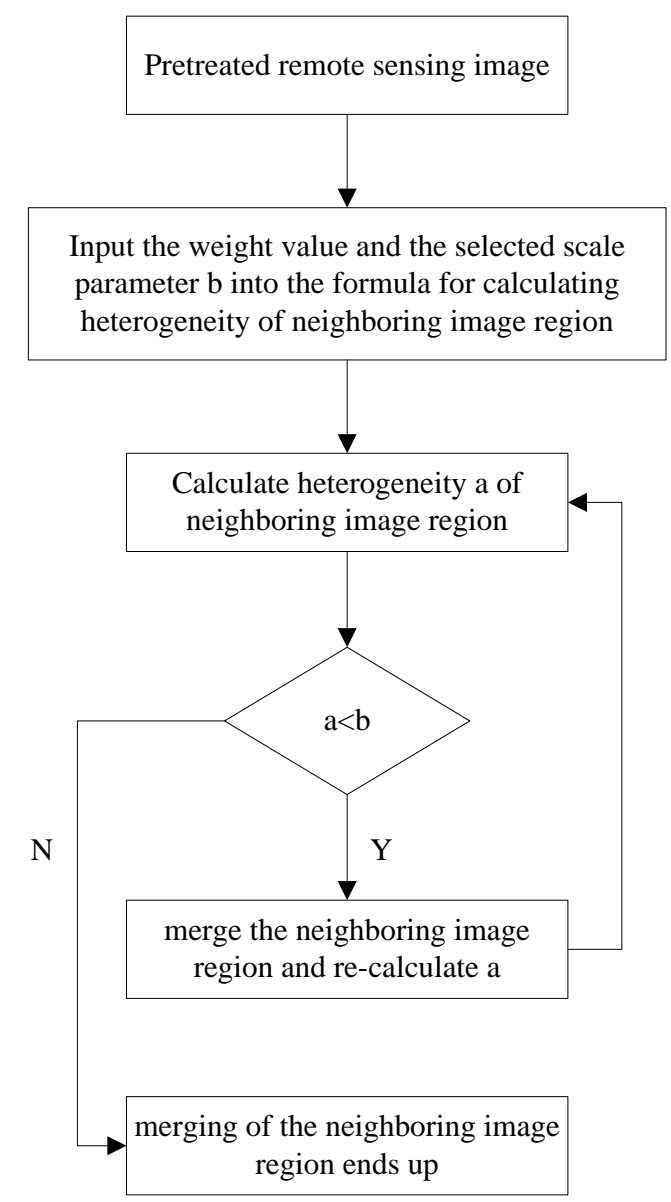

Fig.1 Flow chart of the multi-scale segmentation algorithm

Implementation of the multi-scale segmentation algorithm based on high resolution satellite remote sensing image includes two aspects: selection of scale parameter and heterogeneity calculation of neighboring image region.

\section{A. Selection of the scale parameter}

The scale parameter is selected primarily according to the size of the earth object. The larger scale parameter is setup, the merging is more frequently executed in the algorithm and thus the larger image object is obtained; in the same way, the smaller scale parameter is setup, the merging is less frequently executed in the algorithm and thus the smaller image object is obtained.

\section{B. Heterogeneity calculation of neighboring image region}

Heterogeneity of neighboring image region mainly includes spectrum heterogeneity and shape heterogeneity.

1. Formula for heterogeneity of neighboring image region:

$$
a=w l+(1-w) s
$$

In this formula, a denotes for heterogeneity of neighboring image region; l denotes for spectrum heterogeneity of neighboring image region; s denotes for shape heterogeneity of neighboring image region; w denotes for weight vale and $0 \leq \mathrm{w} \leq 1$
2. Formula for spectrum heterogeneity of neighboring image region:

$$
l=\sum_{\mathrm{i}=1}^{n} \mathrm{w}_{\mathrm{i}} \sigma_{i}
$$

In this formula, 1 denotes for spectrum heterogeneity of neighboring image region; wi denotes for weight value of the $i$ image layer; $\sigma_{i}$ denotes for the spectrum value of the i image layer.

3. Formula for shape heterogeneity of neighboring image region:

$$
\mathrm{s}=w_{1} \times c p+\left(1-w_{1}\right) \times s m
$$

In this formula, s denotes for shape heterogeneity of neighboring image region; $\mathrm{cp}$ denotes for compactness of image region; sm denotes for smoothness of image region; w1 denotes for weight vale and $0 \leq \mathrm{w} \leq 1$.

4. Formula for compactness of neighboring image region:

$$
\mathrm{cp}=\text { len } / \sqrt{\text { num }}
$$

In this formula, len denotes for the real boundary length of image region; num denotes for total number of pixels of image region.

5. Formula for smoothness of neighboring image region:

$$
\mathrm{sm}=\text { len } / L
$$

In this formula, $\mathrm{L}$ denotes for length of rectangular boundary containing the image region.

6. Formula for heterogeneity between larger image object (obtained by merging two smaller neighboring image regions) and its adjacent image region:

$$
a^{\prime}=\omega_{1} l^{\prime}+\left(1-\omega_{1}\right) \mathrm{s}^{\prime}
$$

In this formula, $a^{\prime}$ denotes for heterogeneity of the larger image region; $l$ ' denotes for the spectrum heterogeneity of the larger image region; $S^{\prime}$ denotes for the shape heterogeneity of the larger image region.

\section{IMPLEMENTATION OF ALGORITHM}

A high resolution satellite remote sensing image is selected for classification. As shown in Figure 2, target earth object in image is visually interpreted and found to be covered by various land features in different colors, shapes, categories and sizes.

The novel segmentation algorithm is applied in Figure 2 to perform image segmentation. The segmentation scale is setup to be 20 first and the obtained segmented image is shown in Figure 3. The segmentation scale is setup to be 100 thereafter and the obtained segmented image is shown in Figure 4. 


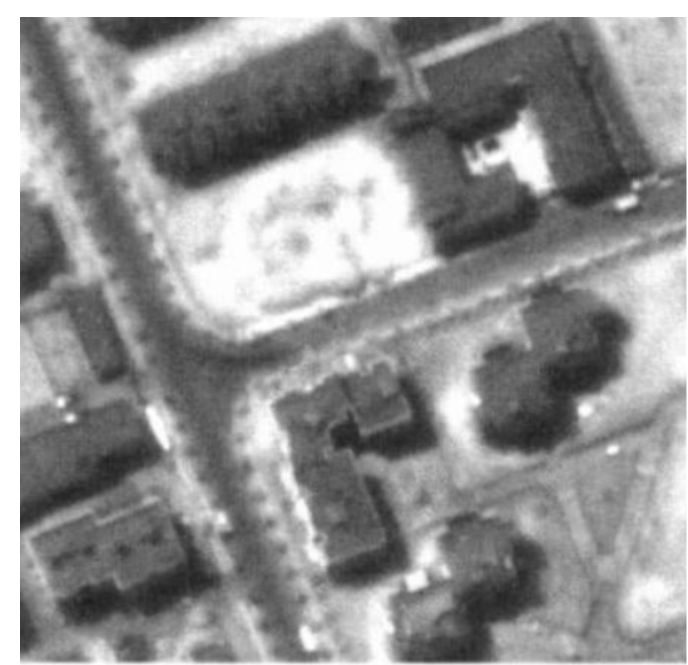

Fig.2 remote sensing image to be segmented

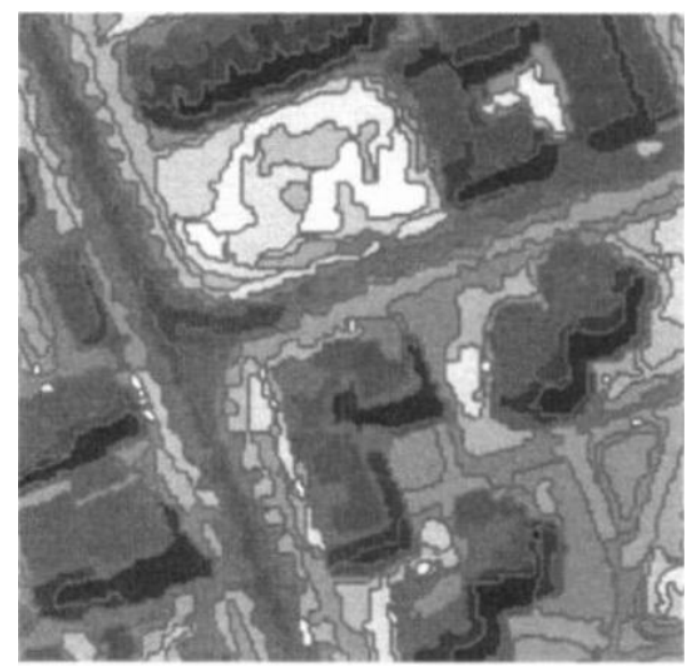

Fig.3 Segmented image with scale 20

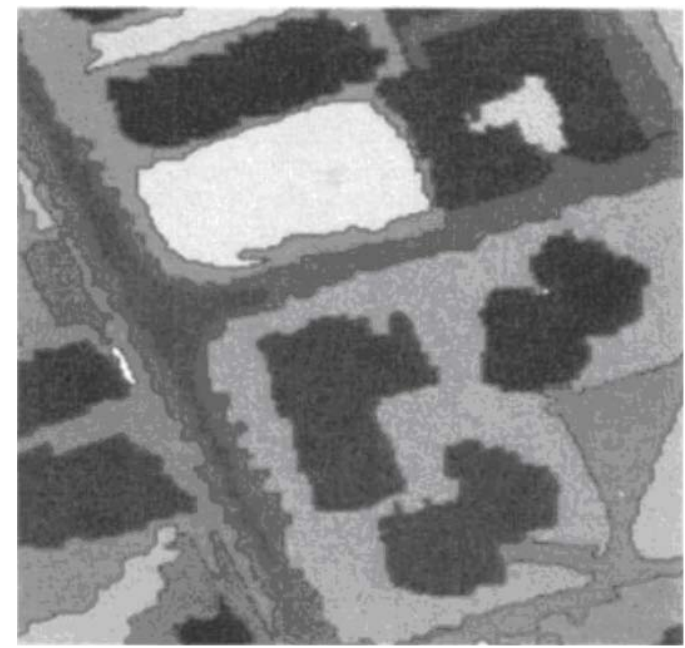

Fig.4 Segmented image with scale 100
From Figure 3 and Figure 4, the following results are obtained:

1. The target earth object is segmented into many image objects. The similar image objects have similar features of spectrum, shape and texture. While dissimilar image objects has significant difference;

2. With the increase of the segmentation scale, the image objects segmented from target earth object become larger;

The above results suggest that multi-scale segmentation algorithm based on high resolution satellite remote sensing image can be used in accurate segmentation of target earth object. In addition, multi-scale segmentation of target earth object can be realized by setting up scale parameter according to the size of the image object.

\section{CONCLUSION}

In this study, we firstly describe the basic ideas, the flow chart and the implementation of the algorithm in great detail. Then the segmentation effect of remote sensing image is verified by experiment. Experiment on multi-scale segmentation algorithm based on high resolution satellite remote sensing image in our study demonstrates that it can display spatial implication of target earth object more faithfully. The results of this study may lay the foundation for following researches on classification and change detection of remote sensing image.

\section{REFERENCES}

[1] Fjortoft R, Gaudin J, Pourthie N, Lalaurie JC, Mallet A, Nouvel JF, "KaRIn on SWOT: Characteristics of near-nadir Ka-band interferometric SAR Imagery,” IEEE Transactions on Geoscience and Remote Sensing, vol.52, pp. 2172- 2185, 2014.

[2] Kharol, S.K., Kaskaoutis, D., Badarinath, K., "Influence of land use/land cover (LULC) changes on atmospheric dynamics over the arid region of Rajasthan state, India,” Journal of Arid Environments, vol.88, pp. 90101, 2013.

[3] R. Duan X, Tian Z, Ding M, Zhao W, "Registration of remote-sensing images using robust weighted kernel principal component analysis," International Journal of Electronics and Communications, vol.67, pp. 20-28, 2013.

[4] Haregeweyn, N., Fikadu, G., Tsunekawa, A., “The dynamics of urban expansion and its impacts on land use/land cover change and small-scale farmers living near the urban fringe: A case study of Bahir Dar, Ethiopia,” Landscape and Urban Planning, vol.106, pp. 149-157, 2012.

[5] Xueliang Zhang, Pengfeng Xiao, Xiaoqun Song, "Boundary-constrained multi-scale segmentation method for remote sensing images,” ISPRS Journal of Photogrammetry and Remote Sensing, vol.78, pp. 15-25, 2013.

[6] Forestier G, Puissant A, Wemmert C, "Knowledge-based region labeling for remote sensing image interpretation,” Computers, Environment and Urban Systems, vol.36, pp. 470-480, 2012. 